\title{
Musculoskeletal pain: prescription of NSAID and weak opioid by primary health care physicians in Sweden 2004-2008 - a retrospective patient record review
}

\author{
This article was published in the following Dove Press journal: \\ Journal of Pain Research \\ 3 August 2010 \\ Number of times this article has been viewed
}

\author{
Metha Brattwall' \\ Ibrahim Turan ${ }^{2}$ \\ Jan Jakobsson ${ }^{3}$ \\ 'Department of Anaesthesia, Institute \\ for Clinical Sciences at Sahlgrenska \\ Academy, Mölndal Hospital, \\ Gothenburg, Sweden; ${ }^{2}$ Foot and Ankle \\ Surgical Centre, Stockholm, Sweden; \\ ${ }^{3}$ Karolinska Institutet, Institution \\ for Physiology and Pharmacology, \\ Department of Anaesthesia, \\ Stockholm, Sweden
}

Purpose: To study the prescription of oral analgesics for musculoskeletal pain by primary care physicians over a 5-year period in Sweden.

Design: A retrospective automatic database review of patient records at four primary health care centers. All prescriptions of NSAIDs, weak opioids, and coprescriptions of gastroprotecting medications to patients with musculoskeletal were retrieved for the period January 1, 2004 to November 11, 2008.

Results: A total of 27,067 prescriptions prescribed to 23,457 patients with musculoskeletal pain were analyzed. Of all prescriptions, NSAIDs were the most commonly prescribed analgesic comprising $79 \%$, tramadol was the second most commonly prescribed analgesic comprising $9 \%$, codeine the third most ( $7 \%$ ), and dextropropoxyphene the fourth $(5 \%)$. The proportion of NSAIDs and weak opioids and the proportion of the different weak opioids prescribed showed no change over time. The proportion of nonselective and selective NSAIDs prescribed changed; Coxib prescriptions decreased from $9 \%$ to $4 \%$ of all analgesics prescribed in 2004-2007 with no change in 2008.

Conclusion: NSAIDs were found to be the dominant class of analgesic prescribed by primary care physicians to patients diagnosed as musculoskeletal pain. No change was observed in the proportion of NSAID and weak opioid prescription over the period studied. Prescription of selective Coxibs decreased and was less than $4 \%$ in 2008. The impact on gastrointestinal and cardiovascular adverse effects associated with the extensive prescription of NSAIDS for musculoskeletal pain warrants further analysis.

Keywords: nonsteroidal anti-inflammatory drugs, tramadol, codeine, dextropropoxyphene, primary care, pattern of use, pharmacoepidemiology

\section{Introduction}

Musculoskeletal pain is one of the most common complaints seen by primary care physicians. ${ }^{1}$ Pain therapy consists of nonpharmacological and pharmacological parts. ${ }^{2}$ The nonsteroidal anti-inflammatory agents (NSAIDs) have become increasingly popular as a second-line therapy. ${ }^{3-5}$ Today, there are a variety of NSAIDs available for prescription: traditional nonselective (tNSAIDs), and the more selective COX-IIinhibitors (Coxibs). The analgesic effects of the different NSAIDs are more or less identical; however, the Coxibs are associated with a lower risk for upper gastrointestinal side effects. ${ }^{6}$ Thus, the Coxibs after their introduction become an alternative to traditional NSAIDs in patients exhibiting risk for upper gastrointestinal bleeding.
Correspondence: Jan Jakobsson Department of Anaesthesia and Intensive Care, Institution for Physiology and Pharmacology, Karolinska Institutet, Stockholm, Sweden Email Jan.Jakobsson@ki.se 
The national pain guidelines provided by the Swedish Council on Technology Assessment in Health Care supports prescription of Coxibs as a cost-effective option in patients with a gastrointestinal risk. ${ }^{2-4}$

There is still a need for individual analysis regarding the risk of side effects including not only the upper but the entire gastrointestinal tract and the cardiovascular system. ${ }^{7-11}$ The long-term use of NSAIDs warrants, however, an in-depth analysis of benefit vs risk on an individual basis.

The aim of this present study was to review the prescription pattern of NSAID and weak opioid analgesics for the treatment of musculoskeletal pain by primary care physicians over a 5-year period with a focus on whether any changes could be detected due to the increasing awareness of the risk for gastrointestinal and cardiovascular risks associated with the long-term systemic use of antiinflammatory drugs.

\section{Study design}

The study protocol was reviewed and approved by the local ethical review board. A retrospective, patient record analysis was performed by a specially designed database tool that automatically reviews the records searching for all diagnostic codes of musculoskeletal pain (ICD-10 codes) and prescriptions codes (ATC-codes) for NSAIDs (nonselective and selective Coxibs) and weak opioids (tramadol, codeine, and dextropropoxyphene) were collected and analyzed. The case records for all patients seen by one of general practitioners, primary care physician, working at four major primary care centers in the Stockholm area, primary care centers of Eriksberg, Curera Söder, Österåker, and Skogås, were reviewed from January 1, 2004 through November 11, 2008.

All data were collected and stored in an anonymous database without any coupling to the individual patient; no further patient details were compiled or stored.

\section{Statistics}

Prescription pattern is presented in absolute numbers and proportion of prescriptions made.

The data are presented for the entire cohort; all prescriptions and patients are further separated into adult patients aged 18-65 years and elderly patients older than 65 years.

Post hoc analysis of annual proportion of prescriptions data was analyzed by Chi-square test; a $P<0.05$ was considered statistically significant.

\section{Results}

A total of 27,067 prescriptions prescribed to 13,641 females (58\%) and 9,906 males (42\%) diagnosed as musculoskeletal pain were reviewed. On the basis of age, 20,800 prescriptions were made to adult patients and 6,267 to elderly patients.

Patients' demographics are presented in Table 1.

There were no major differences in patient demographics or prescription routines between the four primary health care centers studied.

\section{The entire cohort}

NSAIDs were most commonly prescribed analgesic (79\%-80\%) without any change in proportion of prescription over the 5-year period studied. Tramadol was the second

Table I Demographics for the entire cohort studied from January I, 2004 to November II, 2008

\begin{tabular}{|c|c|c|c|c|c|}
\hline & $\begin{array}{l}2004 \\
(N=2909)\end{array}$ & $\begin{array}{l}2005 \\
(N=4849)\end{array}$ & $\begin{array}{l}2006 \\
(N=6 \mid 4 I)\end{array}$ & $\begin{array}{l}2007 \\
(N=5754)\end{array}$ & $\begin{array}{l}2008 \\
(N=3894)\end{array}$ \\
\hline Female, n (\%) & 1766 (60.7) & $2862(59.0)$ & $3523(57.4)$ & 3307 (57.5) & $2183(56.1)$ \\
\hline Male, n (\%) & I I43 (39.3) & I987 (4I.0) & $2618(42.6)$ & $2447(42.5)$ & I7II (43.9) \\
\hline \multicolumn{6}{|l|}{ Age, y } \\
\hline \multicolumn{6}{|l|}{ Total } \\
\hline Mean (SD) & 50.8 (I5.9) & $51.5(16.0)$ & $51.8(16.2)$ & $52.1(16.4)$ & $52.4(16.3)$ \\
\hline Median & 51.0 & 51.0 & 52.0 & 52.0 & 52.0 \\
\hline Min - max & 18-95 & $18-96$ & $|8-10|$ & $18-102$ & 18-98 \\
\hline \multicolumn{6}{|l|}{ Female } \\
\hline Mean (SD) & $51.4(16.0)$ & $52.3(16.1)$ & $52.7(16.3)$ & $52.8(16.6)$ & $53.5(16.3)$ \\
\hline Median & 52.0 & 53.0 & 53.0 & 53.0 & 54.0 \\
\hline Min - max & $18-95$ & $18-96$ & $|8-10|$ & $18-102$ & 18-98 \\
\hline \multicolumn{6}{|l|}{ Male } \\
\hline Mean (SD) & $50.0(15.7)$ & $50.4(15.8)$ & $50.6(15.9)$ & $51.0(16.1)$ & $50.9(16.2)$ \\
\hline Median & 49.0 & 50.0 & 50.0 & 51.0 & 50.0 \\
\hline Min - max & $18-93$ & $|8-9|$ & $18-92$ & 18-93 & $18-94$ \\
\hline
\end{tabular}

Abbreviation: SD, standard deviation. 
most $(9 \%)$ of all analgesics prescribed and comprised $45 \%$ of weak opioid prescriptions without any change over time (44\%-46\%). Codeine, dextropropoxyphene, and selective Coxibs were prescribed in $7 \%, 5 \%$, and $4 \%$ of all prescriptions analyzed (Table 2).

The proportion of NSAID and weak opioid prescriptions in the entire cohort studied did not change during the study period. Prescription of Coxibs decreased, however, between 2004 and $2007(11.5 \%-3.8 \%, P<0.01)$ with no further change in $2007-2008$ (3.9\% of all NSAIDs prescribed). A small reduction in dextropropoxyphene prescriptions was seen: $23 \%-19 \%$ of weak opioid from 2004 to 2008 . Annual numbers of prescriptions are presented in Table 2 and the proportion of prescriptions annually is shown in Figure 1.

\section{Separation of prescription data into adults and elderly patients}

When the prescription data were separated for adult patients and elderly patients, different prescription patterns were observed. NSAIDs were more commonly prescribed to adults compared with elderly patients and weak opioids more commonly to elderly patients $(P<0.05$; Table 3$)$. The proportion of Coxib out of all NSAIDs prescribed was higher among elderly $7 \%$ vs $3 \%(P<0.05$; see Table 4$)$.

Prescription of dextropropoxyphene showed no change among adults but decreased among elderly, compensated by an increase in tramadol prescription (Table 4).

\section{Discussion}

\section{Principal findings}

We found NSAIDs to be commonly prescribed for musculoskeletal pain, and the proportion of NSAIDs and weak opioids prescribed did not change during the 5-year study (2004-2008). The relative proportion of traditional, nonselective NSAIDs and Coxibs prescribed did, however, change: the prescription of traditional nonselective NSAIDs increased and selective Coxibs decreased during the first 4 years studied but with no change during 2007-2008. Prescription of weak opioids was in general more common

Table 2 Annual numbers of prescriptions for the entire patient cohort studied

\begin{tabular}{llllllll}
\hline & $\mathbf{2 0 0 4}$ & $\mathbf{2 0 0 5}$ & $\mathbf{2 0 0 6}$ & $\mathbf{2 0 0 7}$ & $\mathbf{2 0 0 8}$ & Total & $\%$ \\
\hline tNSAIDs & 2,332 & 4,116 & 5,327 & 5,079 & 3,420 & 20,274 & 75 \\
Coxibs & 304 & 289 & 256 & 201 & 140 & 1190 & 4 \\
Codeine & 233 & 384 & 484 & 447 & 314 & 1862 & 7 \\
Dextropropxyphene & 164 & $26 \mathrm{I}$ & 314 & 306 & 164 & 1209 & 5 \\
Tramadol & 317 & 529 & 680 & 612 & 394 & 2532 & 9 \\
All prescriptions & 3,350 & 5,579 & $7,06 \mathrm{I}$ & 6,645 & 4,432 & 27,067 & 100 \\
\hline
\end{tabular}

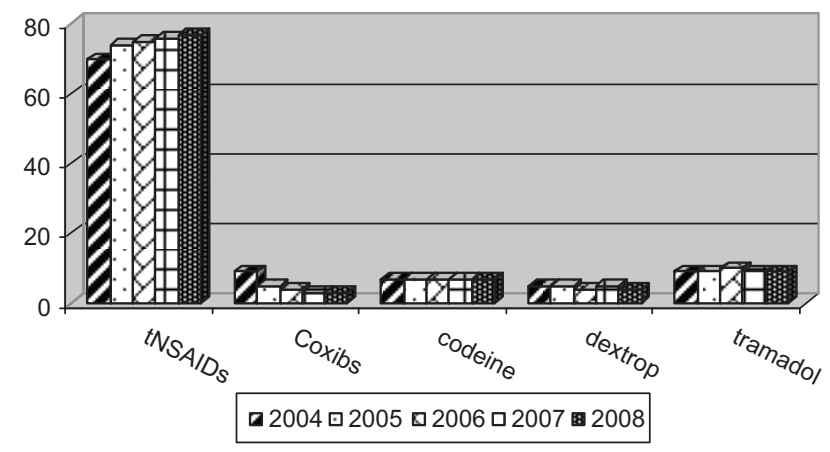

Figure I Proportion of prescriptions from 2004 to 2008 for the entire cohort studied.

among elderly; however, in this more vulnerable patient cohort, no major change in the proportion of NSAIDs and weak opioids was noticed over the time period studied.

\section{Strengths and weaknesses of the study}

The aim of the present survey was to study the prescription pattern of NSAIDs and weak opioids to patients with musculoskeletal pain by primary care physicians. A group of patients commonly seen and where the prescription should be considered not only taking the analgesic effect but also the risks for side effects and health economy into account. ${ }^{12} \mathrm{By}$ the automatic data case record review technique used, a huge number of prescriptions could be analyzed. The prescription pattern was seemingly similar between the four health care centers studied, presenting both urban and suburban environments. It should be acknowledged that no intervention was done; all data were collected retrospectively by an automatic review of patient data records, and thus no doctor bias can by any means be expected. The pattern observed should reasonably present a balanced view of clinical routine prescription practice. The study design carries however limita-

Table 3 Proportion of NSAID and weak opioid prescription among entire cohort and when divided into adults and elderly for the 5 -year study period

\begin{tabular}{llllll}
\hline & $\mathbf{2 0 0 4}$ & $\mathbf{2 0 0 5}$ & $\mathbf{2 0 0 6}$ & $\mathbf{2 0 0 7}$ & $\mathbf{2 0 0 8}$ \\
\hline All & & & & & \\
$\quad$ NSAIDs & 79 & 79 & 79 & 79 & 80 \\
Weak opioids & 21 & 21 & 21 & 21 & 20 \\
$\quad$ All prescriptions & 3,350 & 5,579 & 7,061 & 6,645 & 4,432 \\
Adults & & & & & \\
$\quad$ NSAIDs & 80 & 82 & 82 & 83 & 83 \\
$\quad$ Weak opioids & 18 & 18 & 18 & 17 & 17 \\
$\quad$ All prescriptions & 2,548 & 4,221 & 5,410 & 5,152 & 3,469 \\
Elderly & & & & & \\
$\quad$ NSAIDs & 73 & 70 & 69 & 68 & 72 \\
$\quad$ Weak opioids & 27 & 30 & 31 & 32 & 28 \\
$\quad$ All prescriptions & 802 & 1,358 & 1,651 & 1,493 & 963 \\
\hline
\end{tabular}


Table 4 Proportion of prescriptions in adult patients and elderly patients over the 5 year period studied

\begin{tabular}{llllll}
\hline & $\mathbf{2 0 0 4}$ & $\mathbf{2 0 0 5}$ & $\mathbf{2 0 0 6}$ & $\mathbf{2 0 0 7}$ & $\mathbf{2 0 0 8}$ \\
\hline Adults (age, I8-65 y) & & & & & \\
tNSAIDs & 90 & 95 & 96 & 96.5 & 97 \\
Coxibs & 10 & 5 & 4 & 3,5 & 3 \\
NSAIDs & 100 & 100 & 100 & 100 & 100 \\
No. of prescription & 2,053 & 3,455 & 4,443 & 4,271 & 2,870 \\
Codeine & 34 & 34 & 34 & 34 & 38 \\
Dextropropoxyphene & 19 & 19 & 18 & 19 & 18 \\
Tramadol & 47 & 47 & 48 & 47 & 44 \\
Weak opioids & 100 & 100 & 100 & 100 & 100 \\
No. of prescription & 495 & 766 & 967 & 881 & 599 \\
Elderly (age > 65 y) & & & & & \\
tNSAIDs & 82 & 89 & 93 & 95 & 93 \\
Coxibs & 18 & 11 & 7 & 5 & 7 \\
NSAIDs & 100 & 100 & 100 & 100 & 100 \\
No. of prescription & 583 & 950 & 1,140 & 1,009 & 690 \\
Codeine & 30 & 31 & 30 & 30 & 32 \\
Dextropropoxyphene & 32 & 27 & 28 & 28 & 21 \\
Tramadol & 38 & 41 & 42 & 41 & 47 \\
Weak opioids & 100 & 99 & 100 & 99 & 100 \\
No. of prescription & 219 & 408 & 511 & 484 & 273 \\
\hline
\end{tabular}

tions. The information retrieved included merely prescription information, and no information of comorbidity, concomitant medications, or occurrence of side effects was unfortunately collected. The data retrieved consists only of prescribed medication by ATC code. We have no data about incidence and severity of any side effects, need for extra/emergent health care center consultations, and visits to the emergency department or admission to hospital.

Our findings may not be seen as unexpected. NSAIDs have become the mainstay of therapy for acute musculoskeletal pain., ${ }^{2,3}$ NSAIDs are also commonly recommended as second-line therapy for pain associated with arthritis when paracetamol has been tested or is unable to provide adequate pain control..$^{2-4}$ One should, however, acknowledge that we studied primary care physicians and musculoskeletal pain, in general, and not firmly defined diagnosis of arthrosis or arthritis. Similar studies about prescription pattern for musculoskeletal pain made in the United States and Finland (published in 2001 and 2004) showed variability in prescription routines. ${ }^{13,14}$ Also the findings in a Norwegian survey showed huge variability in patient intake of analgesics for musculoskeletal pain. ${ }^{15}$ These studies describe prescription of NSAID systemic, as well as topical, weak opioids, and also occasionally stronger opioids. The option for over-the-counter (OTC) medication should also be acknowledged. ${ }^{16} \mathrm{We}$ did not retrieve anamnestic information, and we are, thus, not able to state to what extent our patients used OTC drugs. A more recent American survey showed similar findings to ours, ie, a common prescription routine of NSAIDs, ${ }^{17}$ and the proportion of Coxibs prescribed changed with a peak in 2001-2003 followed by a dramatic reduction during 2004. The Vioxx and Bextra retractions following identification of an increased incidence of cardiovascular event in 2004 and 2005 caused a major change in the willingness to prescribe Coxibs. ${ }^{9}{ }^{10}$ Our findings with respect to NSAID use is similar to that found in a nationwide study in Denmark describing the annual NSAID market. ${ }^{18}$ The use of NSAIDs is supported by many national guidelines and international reviews, but the awareness of the risk for side effects associated with NSAIDs and the selective Coxibs have promoted a focus on paracetamol as the baseline drug and provided the option for addition of weak opioids when greater analgesia is desired. ${ }^{19}$

The prescription pattern and compliance to prescribed medication are intrigues parts of the pharmacological management especially in more protracted pain, "chronic pain situations" such as musculoskeletal pain including arthrosis/arthritis. The benefit from the analgesics, reduction of pain, must be evaluated against the risk for side effects in each individual patient. ${ }^{20}$ Cost is also becoming increasingly important, and in many countries, cheapest generic product is recommended in order to reduce direct drug costs. Cost considerations should, however, take into account not only the direct prescribed drug cost but also costs associated with coprescriptions in order to prevent or treat side effects and other resources needed, ie, any additional contacts with health care.$^{21}$ We found a clear switch, increase in prescription of nonselective NSAIDs and a reduction in prescription of Coxibs. The impact of this change needs further attention. The option of paracetamol, topical NSAIDs, and addition of low-dose strong opioid when needed should be considered an alternative.

\section{Unanswered questions and future research}

The aim of the present study was to review prescription pattern and changes over time. Information about dose, dosing, and compliance to prescription was not analyzed. No outcome variables were included; thus, it is not possible to make any assessment of risk vs benefit from the prescribed medications. The risk for gastrointestinal events associated with NASID use is well recognized. The Coxibs were introduced in order to reduce the gastrointestinal risk and the clinical benefit has been well documented. ${ }^{6}$ The beneficial effects with regard to upper gastrointestinal events with the Coxib must, of course, be put into the perspective of the cardiovascular risks. It is, however, becoming increasingly obvious that the cardiovascular risk is 
not class related but differs between the different substances. ${ }^{22,23}$ Further studies analyzing the overall benefit vs risk and pain reduction, incidence and severity of any gastrointestinal and cardiovascular side effects associated with the change in the prescription ratio of traditional NSAIDs (with and without coprescribed gastroprotection) and Coxibs are warranted.

\section{Summary and conclusion}

Anti-inflammatory agents, NSAIDs, were by far the most commonly prescribed analgesic by family physicians for the treatment of musculoskeletal pain. Prescription of Coxibs decreased, however, from 2004 and is only prescribed to a low percentage $(<4 \%)$. The overall proportion between weak opioids (tramadol, codeine, and dextropropoxyphene) and NSAIDs or the relative proportion of the different weak opioids did not change. Studies evaluating the impact of this extensive use of NSAIDs on the upper gastrointestinal, as well as lower, and cardiovascular side effects are thus warranted.

\section{Acknowledgment}

This study has been supported by grants from MSD. All authors have contributed equally in the preparation of this manuscript.

\section{Disclosure}

None of the authors have any competing interests.

\section{References}

1. Hasselström J, Liu-Palmgren J, Rasjö-Wrååk G. Prevalence of pain in general practice. Eur J Pain. 2002;6(5):375-385.

2. Zhang W, Moskowitz RW, Nuki G, et al. OARSI recommendations for the management of hip and knee osteoarthritis, part I: critical appraisal of existing treatment guidelines and systematic review of current research evidence. Osteoarthritis Cartilage. 2007;15(9):981-1000.

3. Zhang W, Moskowitz RW, Nuki G, et al. OARSI recommendations for the management of hip and knee osteoarthritis, Part II: OARSI evidence-based, expert consensus guidelines. Osteoarthritis Cartilage. 2008;16(2):137-162.

4. Weaver AL. Current and emerging treatments for mild/moderate acute ambulatory pain. Am J Ther. 2008;15 Suppl 10:S12-S16.

5. Fendrick AM, Greenberg BP. A review of the benefits and risks of nonsteroidal anti-inflammatory drugs in the management of mild-tomoderate osteoarthritis. Osteopath Med Prim Care. 2009;3:1.

6. Hunt RH, Harper S, Watson DJ, et al. The gastrointestinal safety of the COX-2 selective inhibitor etoricoxib assessed by both endoscopy and analysis of upper gastrointestinal events. Am J Gastroenterol. 2003; 98(8):1725-1733.

Journal of Pain Research

\section{Publish your work in this journal}

The Journal of Pain Research is an international, peer-reviewed, open access, online journal that welcomes laboratory and clinical findings in the fields of pain research and the prevention and management of pain. Original research, reviews, symposium reports, hypothesis formation and commentaries are all considered for publication.
7. Bardou M, Barkun AN. Preventing the gastrointestinal adverse effects of nonsteroidal anti-inflammatory drugs: from risk factor identification to risk factor intervention. Joint Bone Spine. 2010;77(1):6-12.

8. Lanas A, Sopeña F. Nonsteroidal anti-inflammatory drugs and lower gastrointestinal complications. Gastroenterol Clin North Am. 2009;38(2):333-352.

9. Alacqua M, Trifirò G, Cavagna L, et al. Prescribing pattern of drugs in the treatment of osteoarthritis in Italian general practice: the effect of rofecoxib withdrawal. Arthritis Rheum. 2008;59(4):568-574.

10. Barozzi N, Tett SE. What happened to the prescribing of other COX-2 inhibitors, paracetamol and non-steroidal anti-inflammatory drugs when rofecoxib was withdrawn in Australia? Pharmacoepidemiol Drug Saf. 2007;16(11):1184-1191

11. Greenberg JD, Fisher MC, Kremer J, et al. The COX-2 inhibitor market withdrawals and prescribing patterns by rheumatologists in patients with gastrointestinal and cardiovascular risk. Clin Exp Rheumatol. 2009; 27(3):395-401.

12. Drummond M, Ferraz MB, Mason J. Assessing the cost effectiveness of NSAID: an international perspective. J Rheumatol. 1995; 22(7):1408-1411.

13. Mäntyselkä $\mathrm{P}$, Ahonen R, Kumpusalo E, Takala J. Variability in prescribing for musculoskeletal pain in Finnish primary health care. Pharm World Sci. 2001;23(6):232-236.

14. Caudill-Slosberg MA, Schwartz LM, Woloshin S. Office visits and analgesic prescriptions for musculoskeletal pain in US: 1980 vs 2000. Pain. 2004;109(3):514-519.

15. Holtedahl R. Analgesics use in patients with chronic musculoskeletal complaints. Tidsskr Nor Laegeforen. 2004;124(15):1930-1932.

16. Mäntyselkä P, Ahonen R, Viinamäki H, Takala J, Kumpusalo E. Drug use by patients visiting primary care physicians due to nonacute musculoskeletal pain. Eur J Pharm Sci. 2002;17(4-5):201-206.

17. Wilson RD. Analgesic prescribing for musculoskeletal complaints in the ambulatory care setting after the introduction and withdrawal of cyclooxygenase-2 inhibitors. Arch Phys Med Rehabil. 2009;90(4): $1147-1151$

18. Fosbøl EL, Gislason GH, Jacobsen S, et al. The pattern of use of nonsteroidal anti-inflammatory drugs (NSAIDs) from 1997 to 2005: a nationwide study on 4.6 million people. Pharmacoepidemiol Drug Saf. 2008;17(8):822-833.

19. Schnitzer TJ. Update on guidelines for the treatment of chronic musculoskeletal pain. Clin Rheumatol. 2006;25 Suppl 1:S22-S29.

20. Patrignani P, Tacconelli S, Capone ML. Risk management profile of etoricoxib: an example of personalized medicine. Ther Clin Risk Manag. 2008;4(5):983-997.

21. Hur C, Chan AT, Tramontano AC, Gazelle GS. Coxibs versus combination NSAID and PPI therapy for chronic pain: an exploration of the risks, benefits, and costs. Ann Pharmacother. 2006;40(6): $1052-1063$.

22. Combe B, Swergold G, McLay J, et al. Cardiovascular safety and gastrointestinal tolerability of etoricoxib vs diclofenac in a randomized controlled clinical trial (The MEDAL study). Rheumatology. 2009; 48(4):425-432.

23. Farkouh ME, Greenberg BP. An evidence-based review of the cardiovascular risks of nonsteroidal anti-inflammatory drugs. Am J Cardiol. 2009;103:1227-1237.

The manuscript management system is completely online and includes a very quick and fair peer-review system, which is all easy to use. Visit http://www.dovepress.com/testimonials.php to read real quotes from published authors. 